\title{
FRAX $^{\mathrm{TM}:}$ : construindo uma ideia para o Brasil
}

\author{
FRAX ${ }^{\mathrm{TM}:}$ building an idea to Brazil
}

Marcelo de Medeiros Pinheiro', Bruno Muzzi Camargos², Victoria Z. C. Borba ${ }^{3}$, Marise Lazaretti-Castro ${ }^{4}$

\section{RESUMO}

Diferenças genéticas, raciais e antropométricas, bem como da composição corporal, densidade óssea, dieta, atividade física e outros hábitos de vida, contribuem para explicar as divergências na incidência e prevalência de baixa densidade óssea e fraturas em diversos países do mundo. Recentemente, foi desenvolvida uma ferramenta, denominada FRAX ${ }^{\mathrm{TM}}$, para aglutinar os fatores clínicos de risco (genéticos e ambientais) e a densidade óssea, a fim de quantificar a probabilidade de fratura osteoporótica nos próximos dez anos. Em breve, ela será utilizada para indicação de tratamento em pacientes de risco. No entanto, atualmente, está disponível somente para uso em algumas populações que possuem banco de dados prospectivos e consistentes - o que não inclui o Brasil. Este estudo teve o objetivo de fazer uma revisão dos estudos epidemiológicos nacionais e internacionais para melhor compreender peculiaridades e diferenças de fatores clínicos de risco, densidade óssea e probabilidade de fratura entre essas populações. Os autores concluíram que se faz necessária a obtenção de mais dados epidemiológicos representativos da população brasileira para utilização da ferramenta FRAX ${ }^{\mathrm{TM}}$. Para isso, os estudos brasileiros deverão possuir características adequadas, como o delineamento prospectivo, avaliação da qualidade de vida, mortalidade e incapacidade física após as fraturas, levando em consideração a expectativa de vida da população brasileira e a análise de custos diretos e indiretos relacionados às fraturas por osteoporose. À luz do conhecimento atual, a utilização de qualquer um dos bancos de dados das populações, disponibilizadas pelo FRAX ${ }^{\mathrm{TM}}$, não é recomendada no Brasil. Arq Bras Endocrinol Metab. 2009;53(6):783-90

Descritores

Osteoporose; fratura; epidemiologia; FRAX'M ; densidade óssea

\section{ABSTRACT}

Genetic and racial background, body composition, bone mineral density (BMD), diet, physical activity and life style help to explain the wide difference observed in the world prevalence and incidence of osteoporosis. Recently, a fracture assessment tool, named FRAX'M , was developed to integrate clinical risk factors (genetic and environmental conditions) and BMD, in order to quantify the ten-year probability of an osteoporotic fracture. Shortly, it will be used to indicate treatment for high risk patients. However, this tool is now available only to those populations with known reliable and prospective epidemiologic data of the osteoporotic fractures - fact that does not include the Brazilian population. The aim of this paper was to review the main national and international epidemiologic studies to better understand the differences between the clinical risk factors, BMD and fracture probability of these populations. The authors concluded that, to use the FRAX ${ }^{\mathrm{TM}}$ tool, it is necessary more epidemiological data that could characterize the Brazilian population. The future studies should be prospective, evaluate the quality of life, mortality and morbidity after a fracture, as well the life expectancy of the population and the cost-effectiveness and utility related to the osteoporotic fracture. In fact, it is not recommended to use any of the populations available in the FRAX $^{\mathrm{TM}}$ tool, as a substitute for the Brazilian population. Arq Bras Endocrinol Metab. 2009;53(6):783-90

Keywords

Osteoporosis; fracture; epidemiology; FRAX'TM; bone density
1 Disciplina de Reumatologia da Escola Paulista de Medicina da Universidade Federal de São Paulo (Unifesp-EPM), São Paulo, SP, Brasil ${ }^{2}$ Centro de Pós-Graduação da Universidade Federal de Minas Gerais (UFMG), Belo Horizonte, MG, Brasil ${ }^{3}$ Serviço de Endocrinologia e Metabologia do Hospital de Clínicas da Universidade Federal do Paraná (SEMPR), Paraná, PR, Brasil; Sociedade Brasileira para o Estudo do Metabolismo Ósseo e Mineral (Sobemom) ${ }^{4}$ Disciplina de Endocrinologia da Unifesp-EPM; Sociedade Brasileira de Endocrinologia e Metabologia (SBEM), Regiona São Paulo, São Paulo, SP, Brasil

Correspondência para: Marcelo de Medeiros Pinheiro Avenida Dr. Altino Arantes, 669, ap. 105 - Vila Clementino 04042-033 - São Paulo, SP, Brasil mpinheiro@uol.com.br

Recebido em 28/Jan/2009 Aceito em 25/Abr/2009 
Tradicionalmente, a osteoporose é reconhecida como uma doença multifatorial, sendo $70 \%$ desses fatores não modificáveis, isto é, definidos pela carga genética, e $30 \%$ potencialmente modificáveis e, assim, envolvidos com os aspectos ambientais. Dessa forma, um único fator de risco não é capaz de identificar indivíduos com ou sem fratura. Diferenças genéticas, raciais e antropométricas, bem como da composição corporal, densidade óssea, dieta, atividade física e outros hábitos de vida contribuem para explicar as divergências na incidência e na prevalência de baixa densidade óssea e fraturas em diversos países do mundo (1-8).

Os aspectos genéticos desempenham importante influência sobre a aquisição do pico de massa óssea e perda óssea relacionada à idade, bem como na determinação das propriedades estruturais e geométricas do osso. No entanto, a associação entre diferentes polimorfismos gênicos e densidade óssea ou fraturas por osteoporose é bastante controversa e varia em diferentes estudos, inclusive no Brasil (9-14) - fato que pode ser tomado como um provável reflexo das diferenças raciais e ambientais das populações estudadas. Da mesma forma, os fatores ambientais não permitem uniformizar e homogeneizar o impacto de cada um deles em cada região do planeta.

O FRAX ${ }^{\mathrm{TM}}$ é uma ferramenta desenvolvida para aglutinar os fatores clínicos de risco para fratura osteoporótica. Levando em consideração fatores genéticos, ambientais e a densidade óssea do fêmur, objetiva quantificar a probabilidade de fratura por fragilidade óssea nos próximos dez anos. Dessa forma, é possível calcular o risco de o paciente vir a sofrer uma fratura de quadril ou fraturas consideradas maiores, como vértebra, antebraço e úmero. Além disso, o instrumento ilustra bem as peculiaridades e dificuldades acima enunciadas relacionadas à população estudada. Assim, a mudança da população representa importante modificação do risco absoluto de fratura.

Por meio de metanálise, Kanis e cols. (15) avaliaram os dados primários de nove grandes estudos epidemiológicos prospectivos (CaMOS, EVOS/EPOS, DOES, Rotterdam, Sheffield, Dubbo, Hiroshima, Gothenburg e Rochester), com cerca de 59.232 indivíduos no total, dos quais $74 \%$ eram mulheres. Aproximadamente $75 \%$ tinham as medidas da densidade óssea do fêmur e relatos de mais de 5 mil fraturas por fragilidade óssea, como quadril, vertebral clínica, úmero, antebraço e fêmur, contabilizando 249 mil pessoas/ano. Eles analisaram, ainda, outros desfechos, como a qualidade de vida
(Quality-adjusted life years, QALYs) e a mortalidade, de acordo com a expectativa de vida de cada país.

Utilizando cálculos de probabilidade e diversas metodologias, como testes não paramétricos, regressão de Cox, curvas de Kaplan-Meyer/Log Rank, modelos bayesiano e de Poisson e testes de verossimilhança ( $\mathrm{Li}$ kelihood), foi possível avaliar a relevância de cada fator de risco naquele determinado estudo. Os principais fatores clínicos de risco identificados foram: idade, dados antropométricos, fratura prévia, história de fratura de quadril materna ou paterna, tabagismo atual, consumo de álcool, uso de glicocorticosteroides, relato de artrite reumatoide e outras causas secundárias de osteoporose, de acordo com o sexo e a origem étnica. A seguir, foram realizadas revisões sistemáticas de cada um desses fatores de risco, para identificar a consistência das informações e dos achados em diversos cenários populacionais.

Em breve, a Organização Mundial da Saúde (OMS) deverá recomendar a utilização do $\mathrm{FRAX}^{\mathrm{TM}}$ para indicação de tratamento em pacientes de risco, bem como fornecer subsídios para a tomada de decisão por órgãos governamentais e gestores de saúde (16). Com isso, cada país poderá utilizar melhor seus recursos de Saúde, baseando-se nos custos diretos, indiretos e nas QALYs. Até o momento, as populações que podem utilizar-se do FRAX ${ }^{\mathrm{TM}}$ são aquelas que possuem dados epidemiológicos consistentes, como da Áustria, da Alemanha, da Suíça, da China, do Reino Unido, da Itália, da França, da Espanha, da Turquia, do Japão, da Suécia e dos Estados Unidos (caucasianos, pretos, hispânicos e asiáti$\cos$ ). O Brasil, entretanto, ainda não está autorizado a usar essa ferramenta, uma vez que não dispõe de banco de dados prospectivos de referência.

De acordo com os dados do Departamento de População e Indicadores Sociais do Instituto Brasileiro de Geografia e Pesquisa (IBGE) (17), o Brasil possui uma população de mais de 180 milhões de habitantes, com leve predominância feminina, e constituída principalmente por etnia miscigenada, com leve predomínio da raça branca $(53,8 \%)$. Embora não existam grandes estudos epidemiológicos em amostra representativa da população brasileira (18-26), sabe-se que a prevalência dos fatores clínicos de risco para baixa densidade óssea é semelhante aos estudos norte-americanos (27-29) e europeus (3032) (Figura 1). Da mesma forma, é similar no que diz respeito aos fatores clínicos de risco para fratura em estudos internacionais (33-37) e nacionais (8,38-40).

Ao se comparar o banco de dados da densidade mineral óssea para brancos de duas grandes coortes inter- 
nacionais (Study of Osteoporotic Fractures, SOF (4l) e European Vertebral Osteoporosis Study, EVOS (42)) com três estudos nacionais de base populacional (Latin America Vertebral Osteoporosis Study (LAVOS), amostragem de mulheres, acima de 50 anos, de Vitória, Espírito Santo (43); The Sao Paulo Osteoporosis Study (SAPOS), amostragem de mulheres, acima de 40 anos, da região metropolitana de São Paulo, São Paulo (44); e Butantã (amostragem de homens e mulheres, acima de 65 anos, da região metropolitana de São Paulo, São Paulo (45)), observou-se que o LAVOS e SAPOS apresentaram valores mais próximos do EVOS e o estudo Butantã mais semelhante ao SOF (Figura 2, coluna lombar, e Figura 3, colo do fêmur). Uma das razões para explicar

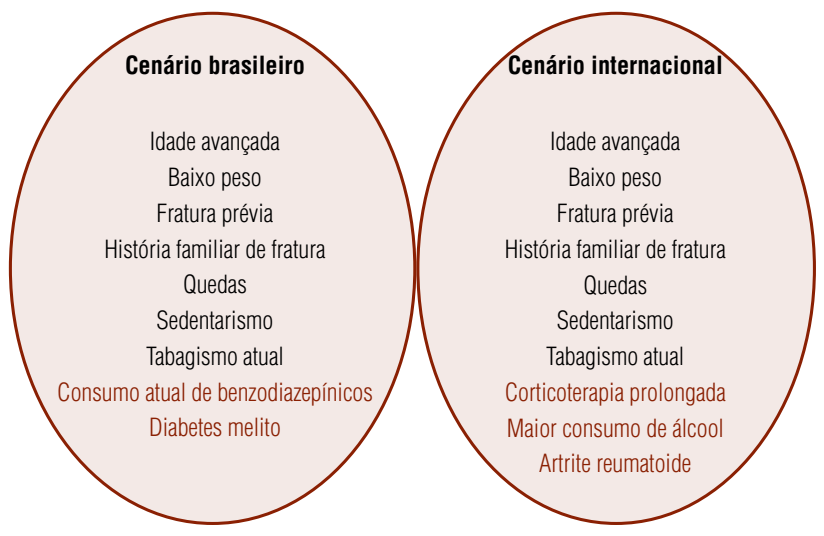

Figura 1. Principais fatores clínicos de risco para osteoporose e fratura em estudos nacionais e internacionais. esses achados discrepantes pode ser o fato de não se ter realizado ajustes estatísticos para a idade e a densidade óssea padronizada (standardized bone mineral density, sBMD), uma vez que os dados originais não estão totalmente disponíveis nas publicações dos estudos internacionais. Dessa forma, vale a pena salientar que pode ter havido um viés de análise decorrente da tecnologia de cada aparelho (fabricantes diferentes), bem como da diversidade de estratificação etária. Esses aspectos podem explicar por que duas amostragens (SAPOS e LAVOS) da população brasileira se aproximaram mais de dados europeus e a outra (Butantã), de valores norte-americanos. Para o planejamento e o delineamento de estudos brasileiros, esses aspectos precisam ser considerados.

De qualquer forma, fica evidente que os brasileiros não possuem grandes diferenças da prevalência dos fatores clínicos de risco nem da densidade mineral óssea da coluna lombar e fềmur proximal quando comparados a populações caucasianas. Por que, então, se possui risco de fratura (Tabela 1 ) tão diferente das populações utilizadas no FRAX ${ }^{\mathrm{TM}}$, como a dos Estados Unidos, Europa, Ásia, Áustria, Alemanha e Suíça (Tabela 2)? O impacto e a relevância dos fatores clínicos de risco (Figura 4), assim como a interação entre eles, particularmente entre sexo, idade, densidade óssea e tipo de fratura, são de fundamental importância epidemiológica. Com isso, há a possibilidade de inúmeras combinações e probabilidades, com um aumento no risco de modo incremental e não de forma geométrica (Tabela 3 ).

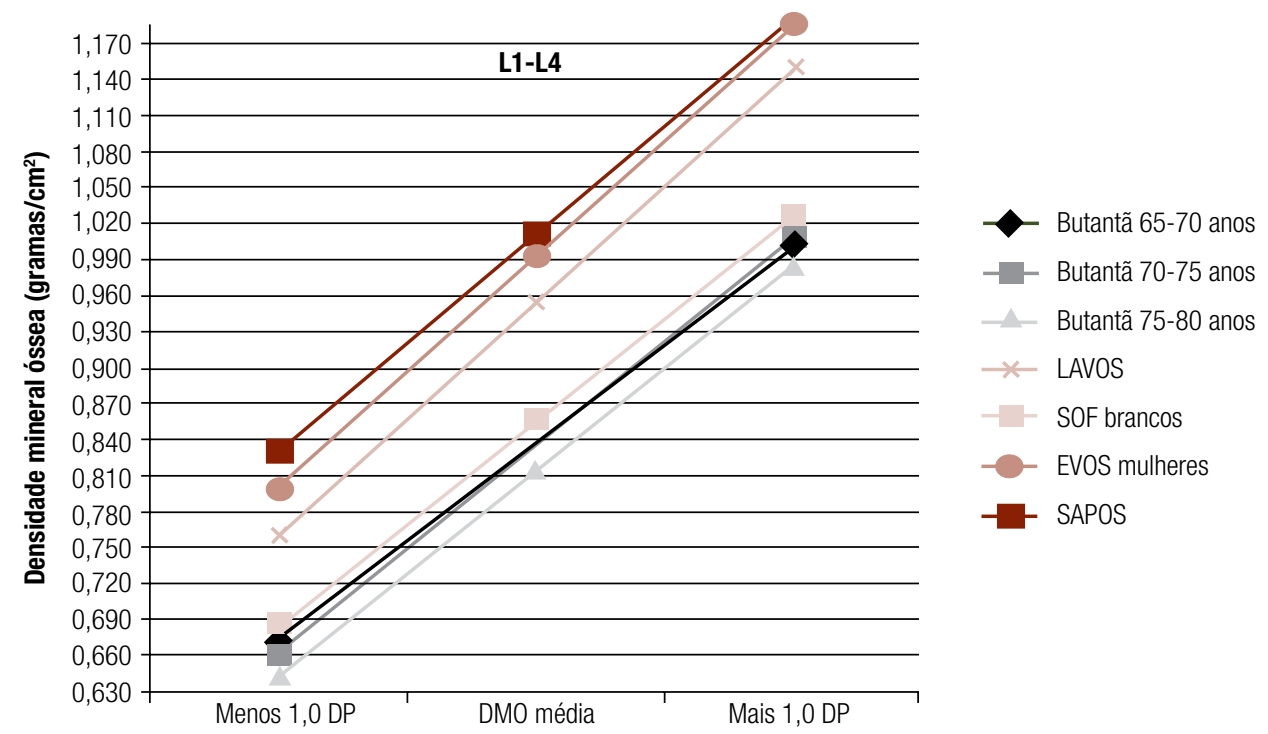

SOF: Study of Osteoporotic Fractures; EVOS: European Vertebral Osteoporosis Study; LAVOS: Latin America Vertebral Osteoporosis Study; SAPOS: Sao Paulo Osteoporosis Study. Ajustes para idade e densidade óssea padronizada (standardized bone mineral density, SBMD) não foram realizados.

Figura 2. Densidade óssea da coluna lombar (L1-L4), de acordo com alguns estudos epidemiológicos brasileiros, em comparação a dados internacionais. 


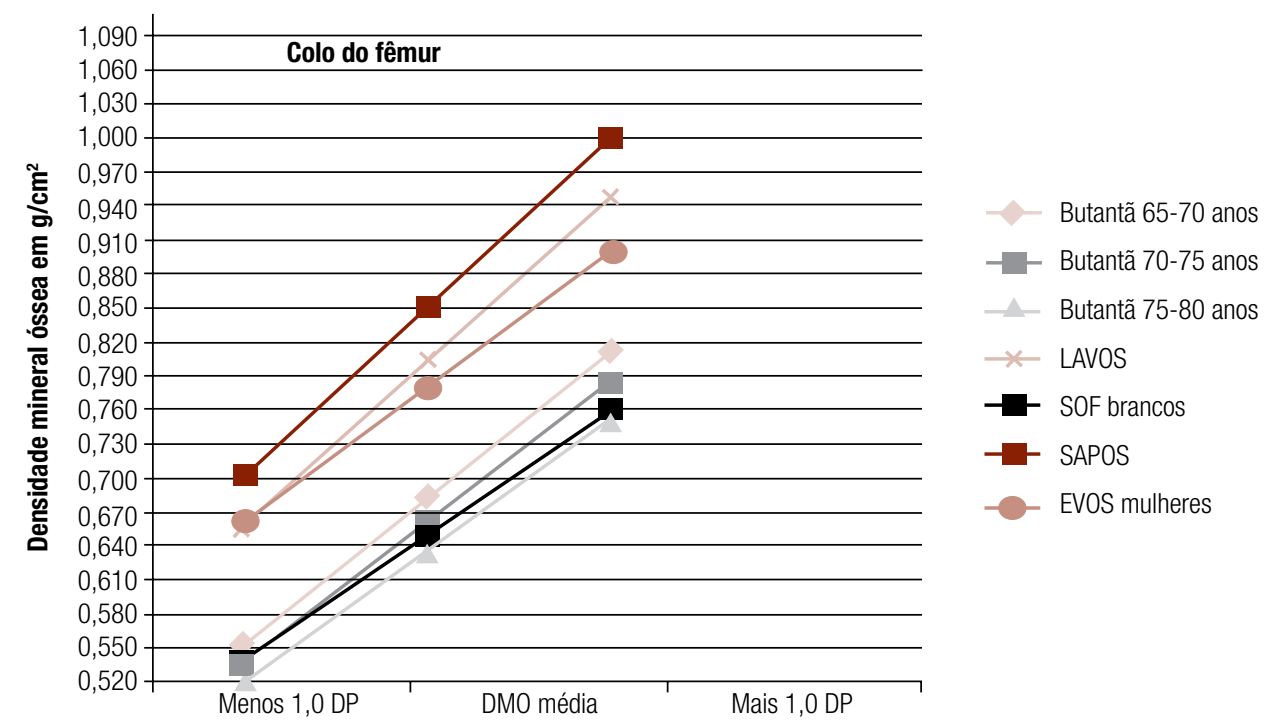

SOF: Study of Osteoporotic Fractures; EVOS: European Vertebral Osteoporosis Study, LAVOS: Latin America Vertebral Osteoporosis Study; SAPOS: Sao Paulo Osteoporosis Study. Ajustes para idade e densidade óssea padronizada (standardized bone mineral density, SBMD) não foram realizados.

Figura 3. Densidade óssea do colo do fêmur, de acordo com alguns estudos epidemiológicos brasileiros, em comparação a dados internacionais.

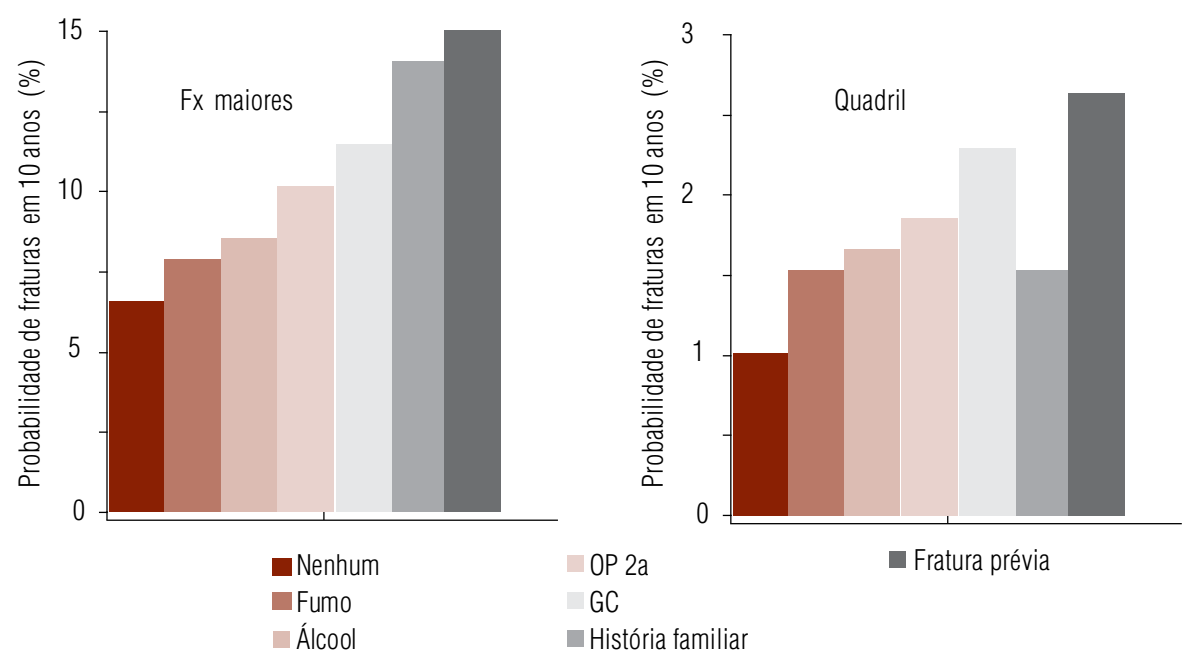

GC: glicocorticosteroides; OP 2a: osteoporose secundária; Fx: fratura.

Figura 4. Influência, impacto e relevância dos fatores clínicos de risco para fraturas maiores e de quadril, conforme a ferramenta FRAXTM.

Tabela 1. Incidência anual de fratura de quadril por 10 mil habitantes, acima de 50 anos de idade, no Brasil

\begin{tabular}{lccc}
\hline Autor/Ano & Região & Mulheres & Homens \\
\hline Sisson de Castro JA, 1990-1992 (1) & Porto Alegre (RS) & 20,2 & 10,5 \\
Castro da Rocha, 1996-2000 (3) & Sobral (CE) & 20,7 & 8,9 \\
Silveira, 2001-2002 (7) & Fortaleza (CE) & 27,5 & 13 \\
Komatsu, 1994-1995 (4) & Marília (SP) & 50 & 18,7 \\
\hline
\end{tabular}

É importante ressaltar, ainda, que a metodologia estatística utilizada para a confecção do instrumento possui, em geral, a peculiaridade de permitir menor poder de generalização ou validação externa dos resultados em populações que não possuam dados epidemiológicos próprios (Tabela 4). Além disso, alguns pontos precisam ser mais bem considerados. Primeiro: os autores 
Tabela 2. Incidência anual de fratura de quadril por 10 mil habitantes, em indivíduos acima de 50 anos, no cenário internacional (1)

\begin{tabular}{lccc}
\hline Cidade, país & Ano & Mulheres & Homens \\
\hline Oslo, Noruega & $1996-1997$ & 118 & 44 \\
Rochester, Estados Unidos & $1988-1989$ & 114 & 41 \\
Fune, Dinamarca & $1973-1975$ & 90 & 30 \\
Wessex, Inglaterra & $1993-1995$ & 82 & 30 \\
Reikjavik, Islândia & $1990-1992$ & 69,7 & 35 \\
Genebra, Suíça & 1991 & 65 & 16 \\
Picardy, França & 1987 & 44 & 18 \\
Hong Kong, China & 1991 & 42,8 & 27 \\
La Plata, Argentina & $1988-1989$ & 33 & 26 \\
Budapeste, Hungria & 1992 & 31,6 & 25 \\
Pequim, China & $1988-1992$ & 9,6 & 10,7 \\
Siena, Itália & $1975-1985$ & 3 & 0,7 \\
\hline
\end{tabular}

Tabela 3. Associação e interação entre os fatores clínicos de risco e o risco de fratura em dez anos para uma mulher de 65 anos de idade, índice de massa corpórea de $25 \mathrm{~kg} / \mathrm{m}^{2}$, de acordo com o número de fatores de risco utilizados pelo instrumento FRAX'M

\begin{tabular}{cc}
\hline Número de fatores de risco & $\begin{array}{c}\text { Probabilidade de fratura de } \\
\text { quadril em 10 anos (\%)* }\end{array}$ \\
\hline 0 & 1,3 \\
1 & $1,7-3,2$ \\
2 & $2,5-6,6$ \\
3 & $3,8-11$ \\
4 & $6,7-17$ \\
5 & 13,24 \\
6 & 30 \\
\hline
\end{tabular}

* Dependência do fator clínico de risco.

\begin{tabular}{l}
\hline Tabela 4. Limitações da ferramenta FRAX ${ }^{\top M}$ \\
\hline Limitações \\
\hline 1. Pequena possibilidade de extrapolação dos dados de outras populações \\
2. Inferência sobre dados não próprios \\
3. Suposição de equações e aproximações e não de verossimilhança \\
4. Modelos população-dependente (influência de cada variável em determinado \\
estudo) \\
5. Comprometimento da confiabilidade dos resultados em estudos não \\
consistentes \\
6. Falta de acesso aos dados populacionais (estritamente matemáticos) \\
\hline
\end{tabular}

usaram os dados de incidência de fratura vertebral do estudo sueco (dados mais consistentes) e extrapolaram para as outras populações. Segundo: o banco de dados da densidade mineral óssea do fềmur é proveniente dos resultados dos indivíduos de 20 a 29 anos provenientes do Third National Health and Nutrition Examina- tion Survey (NHANES III) e foi utilizado em todos os outros estudos. Terceiro: nem todas as causas secundárias relacionadas às fraturas por osteoporose entram no cálculo final do escore. As escolhidas foram apenas hipogonadismo masculino ou feminino (ooforectomia, orquiectomia, anorexia nervosa, panhipopituitarismo e pós-quimioterapia para câncer de mama), doença inflamatória intestinal, imobilização secundária a distúrbios neurológicos (mal de Parkinson, acidente vascular cerebral, distrofia muscular, traumatismo raquimedular e espondilite anquilosante), transplante de órgãos, diabetes melito tipo $\mathrm{l}$ e hipertiroidismo não tratado ou exógeno (tratamento do hipotiroidismo). Quarto: se o valor da densidade óssea é inserido no cálculo, as causas secundárias desempenham menor relevância e, assim, são retiradas do risco final de probabilidade. Quinto: os valores de risco relacionados às causas secundárias acima descritas são muito semelhantes ao risco atribuído à artrite reumatoide isoladamente. Sexto: alguns aspectos não foram incluídos na ferramenta $\mathrm{FRAX}^{\mathrm{TM}}$ ou foram contemplados apenas parcialmente, embora esses fatores estejam associados à perda óssea acelerada e à maior taxa de fraturas vertebrais e não vertebrais (Tabela 5).

Após o cálculo da probabilidade de risco de fratura em dez anos, pode ser estabelecido o limiar para intervenção, baseado na análise de custo-efetividade do tratamento, utilização e distribuição de recursos e expectativa de vida em cada país. Os valores propostos para os Estados Unidos (3\% para quadril e 20\% para fraturas maiores) podem não ser, necessariamente, os mesmos da Inglaterra ou da Suécia, por exemplo. Assim, cada país deve estabelecer seus próprios pontos de corte.

\begin{tabular}{|c|c|}
\hline Não incluídos & Parcialmente contemplados \\
\hline Menopausa precoce & Glicocorticosteroides: dose e duração \\
\hline Terapia hormonal prévia & Artrite reumatoide: tempo e atividade da doença \\
\hline Ingestão de cálcio e vitamina D & $\begin{array}{l}\text { Fratura prévia: número e gravidade das fraturas } \\
\text { vertebrais }\end{array}$ \\
\hline $\begin{array}{l}\text { Marcadores da remodelação } \\
\text { óssea }\end{array}$ & $\begin{array}{l}\text { Causas secundárias: mieloma múltiplo, } \\
\text { hiperparatiroidismo, cirurgia bariátrica, asma, } \\
\text { insuficiência renal crônica, litíase renal, } \\
\text { hipercalciúria idiopática, deficiência de } \\
\text { vitamina D e doença pulmonar obstrutiva } \\
\text { crônica }\end{array}$ \\
\hline Quedas & Tabagismo: anos/maço e hábito prévio \\
\hline Atividade física & História familiar de fratura: avós e irmãos \\
\hline $\begin{array}{l}\text { Tratamentos prévios: tipo e } \\
\text { duração }\end{array}$ & Densidade óssea: coluna lombar \\
\hline
\end{tabular}




\section{SITUAÇÃO DO BRASIL}

A osteoporose desempenha importante problema de Saúde Pública, entretanto, pouco é conhecido sobre a epidemiologia da osteoporose no Brasil. Nos últimos 15 anos, cerca de 250 estudos publicados em revistas com Qualis A internacional foram realizados em nosso país, dos quais 71 (28\%) descreveram a situação epidemiológica atual.

Cerca de $30 \%$ a $40 \%$ das mulheres brancas na pósmenopausa apresentam osteoporose, de acordo com estudos internacionais. No entanto, a prevalência de osteoporose é bastante variável entre os estudos brasileiros, uma vez que possuem tamanho de amostra, critérios de elegibilidade e metodologias diferentes. $\mathrm{Na}$ maioria das vezes, esses trabalhos foram feitos em amostragens de conveniência e em populações de hospitais de atenção terciária, não refletindo, muitas vezes, todas as regiões do Brasil.

O The Brazilian Osteoporosis Study (BRAZOS) é o primeiro estudo epidemiológico realizado em amostra representativa de mulheres e homens brasileiros, com mais de 40 anos de idade, com o objetivo de estimar a prevalência e identificar os principais fatores clínicos de risco associados a fratura por baixo impacto e quedas recorrentes (40). Mais de 2.400 indivíduos (70\% de mulheres) provenientes de todas as regiões do Brasil, incluindo as populações urbana e rural, e de todas as classes socioeconômicas foram avaliados por meio de entrevistas quantitativas, pessoais e domiciliares, de forma aleatória. Esse estudo mostrou que $6 \%$ da população geral adulta relatava ter o diagnóstico de osteoporose. Porém, essa baixa prevalência não deve refletir a realidade, visto que a densitometria óssea não foi realizada. Além disso, houve elevada taxa de fraturas por baixo impacto. Assim, se for utilizada a definição de osteoporose da OMS, na qual o diagnóstico de osteoporose estabelecida pode ser feito na presença de fratura por baixo impacto, teriam-se, pelo menos, $12,8 \%$ de osteoporose em homens e $15,1 \%$ em mulheres.

De acordo com esse estudo, realizado na população geral e não somente na de elevado risco, os principais fatores clínicos de risco para fratura por baixo impacto em mulheres foram idade avançada, história familiar de fratura de fêmur, menopausa precoce, sedentarismo, pior qualidade de vida, maior consumo de fósforo, diabetes melito, uso atual de benzodiazepínicos e quedas recorrentes no último ano. Nos homens foram pior qualidade de vida, tabagismo atual, diabetes melito e sedentarismo.
Recentemente, Clark e cols. (43) demonstraram que a prevalência de fraturas vertebrais morfométricas em cinco países da América Latina foi de 11,18\% (IC95\% $=9,23-13,4)$. Este estudo avaliou 1.922 mulheres com 50 anos ou mais, provenientes do México, da Colômbia, de Porto Rico, da Argentina e do Brasil. No Brasil, foram avaliadas 415 pacientes moradoras da cidade de Vitória, estratificadas aleatoriamente e com ponderação para amostragem populacional. Essa frequência foi semelhante à encontrada em Pequim e em alguns países da Europa, mas menor do que a relatada em estudos norte-americanos que usaram a mesma metodologia. Não houve diferença significante entre os cinco países, mas houve aumento com a idade (de 6,9\%, IC95\% $=4,6-9,1$ em mulheres de 50 a 59 anos para $27,8 \%$, IC95\% $=23,1-32,4$ naquelas com mais de 80 anos; $\mathrm{p}<$ $0,001)$. No modelo final, os fatores de risco significantemente associados com fratura vertebral radiográfica foram a perda relatada de estatura $(\mathrm{OR}=1,63$; IC95\% $1,18-2,25)$ e história de fratura prévia $(\mathrm{OR}=1,52$; IC95\% 1,14-2,03).

Pode-se perceber, portanto, que se faz necessária a obtenção de mais dados epidemiológicos representativos da população brasileira, para se conseguir utilizar a ferramenta FRAX ${ }^{\mathrm{TM}}$. Para tal, os estudos brasileiros deverão possuir características adequadas, como delineamento prospectivo, avaliação da qualidade de vida, mortalidade e incapacidade física após as fraturas, levando em consideração a expectativa de vida de nossa população e a análise de custos diretos e indiretos relacionados com as fraturas por osteoporose. Com isso, pode ser delimitado um valor de custo-efetividade baseado na incidência da doença, com ênfase na prevenção, na hospitalização e no tratamento. Recentemente, três líderes de opinião e presidentes de sociedades brasileiras que estudam a osteoporose (Sociedade Brasileira de Osteoporose, Sobrao), a densitometria (Sociedade Brasileira de Densitometria Clínica, SBDens) e o metabolismo mineral e ósseo (Sociedade Brasileira para o Estudo do Metabolismo Ósseo e Mineral, Sobemom, e Sociedade Brasileira de Endocrinologia e Metabologia, SBEM) conclamaram todas as associações de profissionais relacionados aos temas e propuseram iniciar debates e estudo nacional abrangente (46).

Utilizando as características de pacientes brasileiros, de acordo com os estudos nacionais, realizou-se mais de uma centena de simulações e verificou-se que não é possível utilizar nenhum banco de dados das populações disponibilizadas pelo $\mathrm{FRAX}^{\mathrm{TM}}$, pois os resultados 
foram muito discrepantes. Da mesma forma, não é recomendada a utilização da ascendência étnica do paciente na escolha de sua população de base, como, por exemplo, usar a referência da Itália ou da Espanha, caso $\mathrm{o}$ indivíduo tenha tais origens. Outro aspecto interessante é não indicar o uso dessa ferramenta em pacientes em tratamento para osteoporose, uma vez que não se tem como ajustar o risco pelo efeito da terapêutica.

\section{CONCLUSÃO}

Conhecer a prevalência e os fatores de risco específicos para fraturas osteoporóticas de uma população representa importante estratégia para a instituição de medidas educativas e de promoção de Saúde. No Brasil, um país com grandes dimensões geográficas e enorme variedade étnica, caracterizada por intensa miscigenação e dificuldade para definir cada raça ou até mesmo a cor da pele, mais estudos epidemiológicos são necessários e fundamentais para se desenhar o melhor cenário e o mais próximo da verdadeira realidade nacional. $\mathrm{O}$ conhecimento da prevalência, da incidência e a identificação dos principais fatores clínicos de risco associados à fratura por osteoporose ainda não estão bem definidos e, de maneira geral, têm sido extrapolados de estudos europeus e norte-americanos, o que poderá significar um equívoco. Diante dos estudos apresentados, o reconhecimento do problema e a identificação e inclusão desses fatores de risco na prática médica rotineira poderão auxiliar na avaliação do risco de fraturas e na tomada de decisão individualizada. No entanto, a ferramenta FRAX $^{\mathrm{TM}}$ não pode ser recomendada no Brasil, visto que não se dispõe de dados específicos de custo-efetividade do tratamento para a nossa realidade.

Agradecimentos: aos Departamentos de Epidemiologia Clínica, Bioestatística e de Informática em Saúde (DIS) da Escola Paulista de Medicina da Universidade Federal de São Paulo (UnifespEPM), pela colaboração, assessoria e aconselhamento estatístico para a compreensão dos modelos matemáticos e simulações aplicadas ao cenário brasileiro. Aos médicos pesquisadores Doutora Vera L. Szejnfeld (Unifesp/EPM), Doutora Rosa Maria Pereira Rodrigues (Faculdade de Medicina da Universidade de São Paulo, FMUSP), Doutor Sérgio Ragi-Eis (Centro de Diagnóstico e Pesquisa da Osteoporose do Espírito Santo, Cedoes) e Doutor Cristiano A. Zerbini (Hospital Heliópolis), pela gentileza, disponibilidade e fornecimento dos bancos de dados de densidade mineral óssea dos estudos SAPOS, Butantã, LAVOS e Heliópolis, respectivamente.

Declaração: os autores declaram não haver conflitos de interesse científico neste estudo.

\section{REFERÊNCIAS}

1. Schwartz AV, Kelsey JL, Maggi S, Tuttleman M, Ho SC, Jónsson $\mathrm{PV}$, et al. International variation in the incidence of hip fractures: cross-national project on osteoporosis for the World Health Organization Program for Research on Aging. Osteoporos Int. 1999;9(3):242-53.

2. Siris ES, Miller PD, Barrett-Connor E, Faulkner KG, Wehren LE, Abbott TA, et al. Identification and fracture outcomes of undiagnosed low bone mineral density in postmenopausal women: results from the National Osteoporosis Risk Assessment. JAMA. 2001;286(22):2815-22.

3. Castro da Rocha FA, Ribeiro AR. Low incidence of hip fractures in an equatorial area. Osteoporos Int. 2003;14(6):496-9.

4. Komatsu RS, Ramos LR, SzejnfeldVL. Incidence of proximal femur fractures in Marilia, Brazil. J Nutr Health Aging. 2004;8(5):362-7.

5. Morales-Torres J, Gutierrez-Urena S; Osteoporosis Committee of Pan-American League of Associations for Rheumatology. The burden of osteoporosis in Latin America. Osteoporos Int. 2004;15(8):625-32.

6. Siris ES, Brenneman SK, Miller PD, Barrett-Connor E, Chen YT, Sherwood LM, et al. Predictive value of low BMD for 1-year fracture outcomes is similar for postmenopausal women ages 50-64 and 65 and Older: results from the National Osteoporosis Risk Assessment (NORA). J Bone Miner Res. 2004;19(8):1215-20.

7. Silveira VA, Medeiros MM, Coelho-Filho JM, Mota RS, Noleto JC, Costa FS, et al. Hip fracture incidence in an urban area in Northeast Brazil. Cad Saude Publica. 2005;21(3):907-12.

8. Siqueira FV, Facchini LA, Hallal PC. The burden of fractures in Brazil: a population-based study. Bone. 2005;37(2):261-6.

9. Lazaretti-Castro M, Duarte-de-Oliveira MA, Russo EM, Vieira JG. Vitamin $D$ receptor alleles and bone mineral density in a normal premenopausal Brazilian female population. Braz J Med Biol Res. 1997;30(8):929-32.

10. Hauache OM, Lazaretti-Castro M, Andreoni S, Gimeno SG, Brandão $C$, Ramalho $A C$, et al. Vitamin $D$ receptor gene polymorphism: correlation with bone mineral density in a Brazilian population with insulin-dependent diabetes mellitus. Osteoporos Int. 1998;8(3):204-10.

11. Ramalho AC, Lazaretti-Castro M, Hauache O, Kasamatsu T, Brandão $C$, Reis $A F$, et al. Fractures of the proximal femur: correlation with vitamin D receptor gene polymorphism. Braz J Med Biol Res. 1998;31(7):921-7.

12. Barros ER, Kasamatsu TS, Ramalho AC, Hauache OM, Vieira JG, Lazaretti-Castro M. Bone mineral density in young women of the city of São Paulo, Brazil: correlation with both collagen type I alpha 1 gene polymorphism and clinical aspects. Braz J Med Biol Res. 2002;35(8):885-93.

13. Heilberg IP, Teixeira SH, Martini LA, Boim MA. Vitamin D receptor gene polymorphism and bone mineral density in hypercalciuric calcium-stone-forming patients. Nephron. 2002;90(1):51-7.

14. Heilberg IP, Hernandez E, Alonzo E, Valera R, Ferreira LG, Gomes $\mathrm{SA}$, et al. Estrogen receptor (ER) gene polymorphism may predict the bone mineral density response to raloxifene in postmenopausal women on chronic hemodialysis. Ren Fail. 2005;27(2):155-6.

15. Kanis JA, Johnell O, Oden A, Johansson H, McCloskey E. FRAX and the assessment of fracture probability in men and women from the UK. Osteoporos Int. 2008;19(4):385-97.

16. Kanis JA [Internet]. On behalf of the World Health Organisation Scientific Group. Available from: http://www.shef.ac.uk/FRAX/reference.htm.

17. IBGE [Internet]. Censo Demográfico 2000: características da população e dos domicílios: resultados do universo. Disponível em: http://www.ibge.gov.br/home/estatistica/populacao/censo2000/ default.shtm 
18. Szejnfeld VL, Atra E, Baracat EC, Aldrighi JM, Civitelli R. Bone density in white Brazilian women: rapid loss at the time around the menopause. Calcif Tissue Int. 1995;56 (3):186-91.

19. Zerbini CA, Latorre MR, Jaime PC, Tanaka T, Pippa MG. Bone mineral density in Brazilian men 50 years and older. Braz J Med Biol Res. 2000;33(12):1429-35.

20. Guimarães SVM, Fuchs SC, Vanni BF, Silva BH, Castro JAS. Predictors of total body bone mass in pre-menopausal women of Southern Brazil: a population-based study. Reprod Clim. 2002;17(2):112-7.

21. Lanzillotti HS, Lanzillotti RS, Trotte AP, et al. Osteoporosis in postmenopausal women, dietary calcium and other risk factors. Rev Nutr. 2003;16(2):181-93.

22. Costa-Paiva L, Horovitz AP, Santos AO, Oliveira A, Fonsechi-Carvasan GA, Pinto-Neto AM, et al. Prevalence of osteoporosis in postmenopausal women and association with clinical and reproductive factors. Rev Bras Ginecol Obstet. 2003;25(7):507-12.

23. Camargo MB, Cendoroglo MS, Ramos LR, de Oliveira Latorre Mdo R, Saraiva GL, Lage A, et al. Bone mineral density and osteoporosis among a predominantly Caucasian elderly population in the city of São Paulo, Brazil. Osteoporos Int. 2005;16(11):1451-60.

24. Sen SS, Rives VP, Messina OD, Morales-Torres J, Riera G, AnguloSolimano JM, et al. A risk assessment tool (OsteoRisk) for identifying Latin American women with osteoporosis. J Gen Intern Med. 2005;20(3):245-50.

25. Frazão $P$, Naveira $M$. Factors associated with low bone mineral density among white women. Rev Saude Publica. 2007;41(5):740-8.

26. Steiner ML, Fernandes CE, Strufaldi R, Azevedo LH, Stephan $C$, Pompei LM, et al. Accuracy study on "Osteorisk": a new osteoporosis screening clinical tool for women over 50 years old. Sao Paulo Med J. 2008;126(1):23-8.

27. Black DM, Palermo L, Pearson J, Abbott T, Johnell O. A simple, useful risk factor system can identify the large majority of women with osteoporosis. Bone. 1998;23(Suppl. 5):605

28. Lydick E, Cook K, Turpin J, Melton M, Stine R, Byrnes C. Development and validation of a simple questionnaire to facilitate identification of women likely to have low bone density. Am J Manag Care. 1998;4(1):37-48.

29. Cadarette SM, Jaglal SB, Kreiger N, Mclsaac WJ, Darlington GA, Tu JV. Development and validation of the Osteoporosis Risk Assessment Instrument to facilitate selection of women for bone densitometry. CMAJ. 2000;162(9):1289-94.

30. Michaëlsson K, Bergström R, Mallmin H, Holmberg L, Wolk A, Ljunghall S. Screening for osteopenia and osteoporosis: selection by body composition. Osteoporos Int. 1996;6(2):120-6.

31. Sedrine WB, ChevallierT, Zegels B, Kvasz A, Micheletti MC, Gelas $B$, et al. Development and assessment of the Osteoporosis Index of Risk (OSIRIS) to facilitate selection of women for bone densitometry. Gynecol Endocrinol. 2002;16(3):245-50.

32. Salaffi F, Silveri F, Stancati A, Grassi W. Development and validation of the osteoporosis prescreening risk assessment (OPERA) tool to facilitate identification of women likely to have low bone density. Clin Rheumatol. 2005;24(3):203-11.

33. Black DM, Steinbuch M, Palermo L, Dargent-Molina P, Lindsay R, Hoseyni MS, et al. As assessment tool for predicting fracture risk in postmenopausal women. Osteoporos Int. 2001;12(7):519-28.

34. Dargent-Molina P, Douchin MN, Cormier C, Meunier PJ, Bréart G; EPIDOS Study Group. Use of clinical risk factors in elderly women with low bone mineral density to identify women at higher risk of hip fracture:The EPIDOS prospective study. Osteoporos Int. 2002;13(7):593-9.

35. Papaioannou A, Joseph L, loannidis G, Berger C, Anastassiades T, Brown JP, et al. Risk factors associated with incident clinical vertebral and nonvertebral fractures in postmenopausal women: the Canadian Multicentre Osteoporosis Study (CaMos). Osteoporos Int. 2005;16(5):568-78.

36. Henry MJ, Pasco JA, Sanders KM, Nicholson GC, Kotowicz MA. Fracture Risk (FRISK) Score: Geelong Osteoporosis Study. Radiology. 2006;241(1):190-6.

37. Siris ES, Brenneman SK, Barrett-Connor E, Miller PD, Sajjan S, Berger ML, et al. The effect of age and bone mineral density on the absolute, excess, and relative risk of fracture in postmenopausal women aged 50-99: results from the National Osteoporosis Risk Assessment (NORA). Osteoporos Int. 2006;17(4):565-74.

38. Ramalho AC, Lazaretti-Castro M, Hauache O, Vieira JG, Takata E, Cafalli $F$, et al. Osteoporotic fractures of proximal femur: clinical and epidemiological features in a population of the city of São Paulo. Sao Paulo Med J. 2001;119(2):48-53.

39. Pinheiro MM, Castro CH, Frisoli A Jr, Szejnfeld VL. Discriminatory ability of quantitative ultrasound measurements is similar to dual-energy X-ray absorptiometry in a Brazilian women population with osteoporotic fracture. Calcif Tissue Int. 2003;73(6):555-64.

40. Pinheiro MM, Ciconelli RM, Martini LA, Ferraz MB. Clinical risk factors for osteoporotic fractures in Brazilian women and men: the Brazilian Osteoporosis Study (BRAZOS). Osteoporos Int 2009;20(3):399-408.

41. Cummings SR, Black DM, Nevitt MC, Browner W, Cauley J, Ensrud $\mathrm{K}$, et al. Bone density at various sites for prediction of hip fractures. The Study of Osteoporotic Fractures Research Group. Lancet. 1993;341(8837):72-5.

42. Lunt M, Felsenberg D, Adams J, Benevolenskaya L, Cannata J Dequeker $\mathrm{J}$, et al. Population-based geographic variations in DXA bone density in Europe: the EVOS Study. European Vertebral Osteoporosis. Osteoporosis Int. 1997;7(3):175-89.

43. Clark P, Cons-Molina F, Deleze M, Ragi S, Haddock L, Zanchetta JR, et al. The prevalence of radiographic vertebral fractures in Latin American countries: the Latin American Vertebral Osteoporosis Study (LAVOS). Osteoporos Int. 2009;20(2):275-82.

44. Pinheiro MM, Szejnfeld VL. Comunicação pessoal, 2008.

45. Pereira RM. Comunicação pessoal, 2008.

46. Lazaretti-Castro M, Eis SR, Marques Neto JF. A prevenção da osteoporose levada a sério: uma necessidade nacional. Arq Bras Endocrinol Metab. 2008;52(4):712-3. 